\title{
Treatment of Acne Vulgaris With Salicylic Acid Chemical Peel and Pulsed Dye Laser: A Split Face, Rater-Blinded, Randomized Controlled Trial
}

\author{
Olga Lekakh ${ }^{1 *}$, Anne Marie Mahoney ${ }^{2}$, Karlee Novice, Julia Kamalpour², Azeen Sadeghian ${ }^{3}$, \\ Dana Mondo ${ }^{2}$, Cathy Kalnicky ${ }^{4}$, Rong Guo ${ }^{4}$, Anthony Peterson², Rebecca Tung ${ }^{2}$ \\ ${ }^{1}$ Loyola University Chicago Stritch School of Medicine, Maywood, IL 60153, USA \\ ${ }^{2}$ Loyola University Chicago Stritch School of Medicine, Department of Medicine, Division of Dermatology, Maywood, \\ IL 60153, USA \\ ${ }^{3}$ Louisiana State University School of Medicine, Department of Dermatology, New Orleans, LA 70112, USA \\ ${ }^{4}$ Loyola University Chicago Stritch School of Medicine, Division of Health Sciences, Maywood, IL 60153, USA
}

\section{*Correspondence to}

Olga Lekakh, BS; Loyola University Medical Center, Division of

Dermatology 2160 S. First Avenue

Bldg. 54, Room 101 Maywood, IL 60153, USA

Tel: +708-2166533;

Fax: +708-2162444

Email: olekakh@luc.edu

Published online 27 October 2015

\begin{abstract}
Introduction: Pulsed dye laser (PDL) has been used to treat acne lesions and scar erythema by interrupting superficial vasculature. Salicylic acid chemical peels are employed chiefly due to their lipophilic, comedolytic, and anti-inflammatory properties. Although studies have looked at peels and laser therapy independently in acne management, we examined these treatments in combination. Our primary objective was to evaluate the safety and efficacy of concurrent use of salicylic acid peels with PDL versus salicylic acid peels alone in the treatment of moderate to severe acne vulgaris.

Methods: Adult patients with moderate to severe acne were included. Subjects received a total of 3 treatments at 3-week intervals. Per randomized split-face treatment, at week 0 , one half of the subject's face was treated with PDL $(595 \mathrm{~nm})$ followed by whole face application of a $30 \%$ salicylic acid peel. At weeks 3 and 6, the treatments were repeated. At 0 and 9 weeks, patients were assessed with the Global Evaluation Acne (GEA) scale and Dermatology Life Quality Index (DLQI) questionnaire.

Results: Nineteen subjects were enrolled, and 18 completed the study. Significant improvement in acne was seen in both the combined (laser and peel) and chemical peel alone treatment arms $(P<.0005$ and $P=.001)$. Using the GEA scale score, compared to week 0 , the mean difference in acne improvement at week 9 was -1.61 in the combination therapy group versus -1.11 in the peel only group. Based on the GEA scale scoring, a statistically significant greater difference in acne improvement was seen, from week 0 to week 9 , in the combination treatment group compared with the peel only group $(P=.003)$. Conclusion: While acne subjects had significant benefit from the salicylic acid peel alone, they experienced greater significant benefit from PDL treatment used in conjunction with salicylic acid peels. The adjunctive utilization of PDL to salicylic acid peel therapy can lead to better outcomes in acne management.
\end{abstract}

Keywords: Lasers; Acne; Combination therapy; Multimodal treatment.

\section{Introduction}

Acne vulgaris is a chronic inflammatory disorder of the pilosebaceous unit that is associated with significant psychosocial repercussions. ${ }^{1-3}$ The elements of acne pathophysiology-follicular epidermal hyperproliferation, androgen-induced increased sebum production, inflammation, and bacterial colonization of hair follicles by Propionibacterium acnes - are the main targets for acne treatment modalities. Acne lesions most frequently occur on the face, chest, and back, and can cause severe inflammation and scarring that often carries negative social stig- mata and can lead to impairment of quality of life. ${ }^{1,4-6}$ Acne treatment options vary depending on the severity of the disease. Mild acne is often treated with topical retinoids, benzoyl peroxide, or topical antibiotics; moderate acne treatment usually includes the addition of oral antibiotics or oral contraceptives; severe acne often necessitates implementation of systemic medications such as anti-androgens like spironolactone or isotretinoin. ${ }^{1,5,7}$ Current frontiers of investigation in acne research include the adjuvant use of phototherapy, lasers, and chemical peels with traditional therapy. ${ }^{8}$ 
Pulsed dye laser (PDL) is a flash-lamp excited pulse dye laser which delivers a high-energy beam of yellow light at a wavelength and duration that has been optimized for the selective treatment of vascular lesions. PDL has been FDA indicated for treating several benign cutaneous vascular lesions as well as inflammatory acne vulgaris., ${ }^{9,10}$ With regard to acne, PDL targets blood vessels in the dermis and resolves redness from small broken capillaries found in new acne scars. ${ }^{10}$ There is also evidence that PDL can impact both active inflammatory and non-inflammatory acne lesions via alteration of the cutaneous cytokine milieu, particularly by increasing TGF-beta, which is immunosuppressive and inhibits keratinocyte proliferation. Low fluences have been shown to stimulate collagen production which can help with acne scarring. ${ }^{11-13}$

The use of various superficial chemical peels, particularly the agent salicylic acid (SA), have been found to be efficacious in the treatment of acne with superficial peels. ${ }^{14-17}$ SA peels soften the stratum corneum and cause skin shedding by loosening the intracellular matrix and corneocyte connections which can lead to an improvement in non-inflammatory comedones. ${ }^{18} \mathrm{SA}$ also inhibits the arachidonic acid cascade leading to a decrease in inflammatory lesions. ${ }^{19}$ Similar to PDL, SA peels have a low side effect profile and are often used as a treatment modality for acne. Despite extensive usage in clinical practice, there have been relatively few trials demonstrating the efficacy of SA peeling regimens in the treatment of acne.

Although there are clinical studies in the literature investigating SA peels and PDL independently in the treatment of acne, we chose to examine the use of these modalities as a combined treatment. The objective of this study was to evaluate the safety and efficacy of concurrent use of SA peels with PDL versus salicylic peels alone for the treatment of moderate to severe acne vulgaris. Our hypothesis is that SA peels used in conjunction with PDL will be more effective in treating acne than SA peels alone.

\section{Methods}

Subject Population

This study was performed at the Loyola University Health System, Division of Dermatology in LaGrange Park, IL. Subjects were aged 18 and older. Inclusion criteria included subjects in good health with Fitzpatrick skin type I, II, or III, and baseline moderate to severe acne vulgaris as defined by grades 3, 4, or 5 on the Global Evaluation Acne (GEA) scale. Exclusion criteria included pregnancy or lactation, current smokers, previous or current isotretinoin treatment, cosmetic procedures within 3 months of enrollment in the study, active infection on the face excluding acne, allergy to salicylates or petroleum jelly, and a history of bleeding disorders.

\section{Study Design}

The study was a randomized, rater blinded, split-face prospective clinical trial. Subjects received a total of 3 treatments at 3-week intervals. At the baseline visit, prior to the first treatment, subjects were consented and a coin flip was used to randomly determine which side of the subject's face would be treated with PDL. A result of "head-side up" on the coin resulted in the right side being treated, while "tails" on the coin resulted in the left side being treated. At the treatment visits occurring at weeks 0 , 3 and 6 , the subject's face was initially cleansed with $70 \%$ alcohol. Afterwards, half of the subject's face was treated with laser, utilizing the PDL (595 nm) [VBeam Perfecta, Syneron-Candela Inc, Irvine, CA] at laser settings of 7 $\mathrm{mm}$ spot size, energy 10 Joules, 10 millisecond pulse duration, cooling setting 2. Finally, two coats of a 30\% SA peeling solution [Delasco Dermatologic Lab and Supply, Council Bluffs, IA] (with large cotton-tipped applicators) were applied to the subject's entire face and remained in place for 3-5 minutes. Once a white crystallization appeared, cool washcloths were applied for subject comfort, and the face was wiped clean with water. Triamcinolone acetonide $(0.1 \%)$ was then applied to the entire face. Subjects returned for a final clinical evaluation at week 9 . The same dermatologist performed all procedures to avoid inter-physician variability in technique.

\section{Clinical Evaluation}

The GEA scale was used for grading acne severity, which has been shown to have good inter-rater reliability. ${ }^{20}$ It consists of a visual analog scale ranging from 0-5 ("clear to very severe acne") in the clinical assessment of acne severity.

At baseline, subjects were photographed and their Fitzpatrick Skin Type was recorded. At the weeks 0 and 9 visits, patients were photographed and a blinded clinician used the GEA acne evaluation scales to numerically $(0-5)$ grade each side of the patient's face. The photographs were taken with a digital, high-resolution camera under standardized conditions for distance (same f-stop throughout procedure) and lighting.

\section{Subject's Self-Assessment}

At the weeks 0 and 9 visits, patients completed the Dermatology Life Quality Index (DLQI) questionnaire which is a simple 10-question dermatology-specific quality of life questionnaire that is widely used in dermatology clinical trials. $^{21}$

\section{Statistical Analyses}

All statistical analysis was performed using SPSS for Windows (SPSS 18.0, IBM) and significance was determined where $P<.05$. The paired samples $t$ test was used to compare the subject's pre-treatment GEA score at week 0 to the subject's post-treatment GEA score at week 9. McNemar's test for paired samples was used to compare the subject's pre-treatment self-assessment DLQI questionnaire at week 0 to the subject's post-treatment assessment at week 9.

\section{Results}

Nineteen patients were enrolled, and 18 completed the study. There was one dropout secondary to time commit- 
ment issues. Of the 18 patients who completed the study, there were 14 females and 4 males, with an average age of 26.3 years old with a standard deviation of 11.2, ranging from 18 to 52 years old. At week 0 and week 9 , the mean GEA score was calculated for the side of the face treated with the SA peel alone and for the side of the face treated with combination treatment of the salicylic peel and PDL. For the combined treatment side, the mean GEA acne score was 2.72 at week 0 , and 1.11 at week 9 , resulting in an overall decrease of 1.61 on the GEA scale. For the side of the face treated with peel alone, the mean GEA acne score was 2.67 at week 0 , and 1.56 at week 9 , resulting in an overall decrease of 1.11 (Figure 1).

Based on the GEA scale, there was a statistically significant improvement in acne, from week 0 to 9 , for both the SA peel alone $(P=.001)$ and combination treatment with SA peel and PDL $(P<.0005)$. More importantly, there was a statistically significant difference, from week 0 to 9 , between the combination treatment with the SA peel and PDL and peel alone $(P=.003)$ (Figure 2).

\section{Discussion}

Prior studies investigating the use of PDL in the treatment of moderate acne have shown varying degrees of decreased inflammation. ${ }^{14,22,23}$ Seaton et al performed a randomized controlled trial which suggested that one session of PDL (585 nm) improved inflammatory facial acne for up to twelve weeks with no major side effects. The total lesion counts fell by $53 \%$ and inflammatory lesion counts were $49 \%$ lower. $^{24}$ Another study, comparing PDL (585 $\mathrm{nm}$ ) to a combination 585/1,064 nm laser (sequential dual wavelength PDL) in a 12 week split-face comparison of 16 subjects, showed reductions of inflammatory acne lesions of $86 \%$ on the PDL side and $89 \%$ on the $585 / 1,064 \mathrm{~nm}$ side after three treatments performed at 2 week increments. ${ }^{25}$ We demonstrated that concurrent use of SA and PDL for the treatment of acne is both efficacious and safe. While the results show that there was improvement with SA alone, we proved our hypothesis true by showing that the combination intervention was found to be significantly

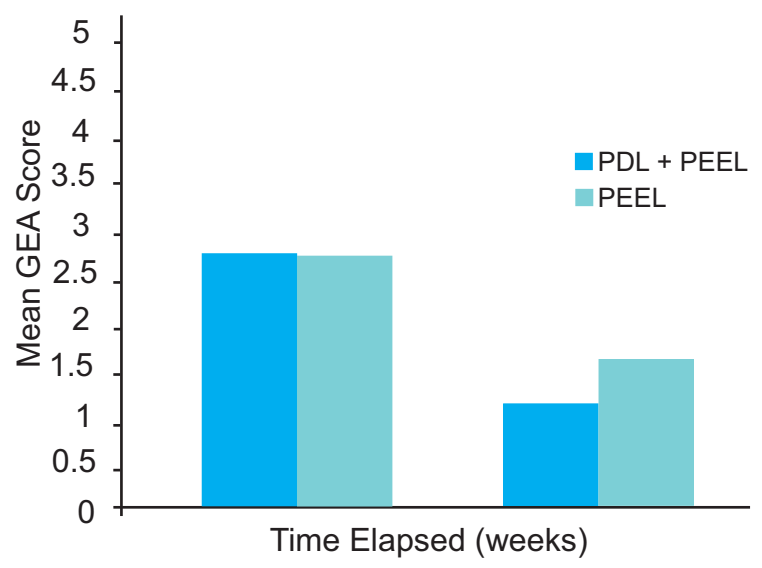

Figure 1. GEA acne evaluation results. At week 9, PDL treatment combined with the SA peel reduced the average GEA 1.61 points, and the SA peel alone reduced the GEA 1.11 points. more efficacious. There was a mean decrease in the GEA scale of 1.61 for the combined side versus 1.11 for the SA side alone. The enhanced efficacy seen with the addition of PDL is likely related to its multi-faceted mechanism of action in acne; this includes an impact on active acne lesions, both inflammatory and non-inflammatory via increased TGF-beta, and a decrease in post inflammatory erythema and induction of neocollagenesis for acne scars. ${ }^{19}$ In addition to the clinical efficacy, patients reported a statistically significant improvement in their level of self-consciousness about their acne at the end of the study compared to week 0 . There were no reported or observed minor or serious adverse events in either treatment arm. Our study demonstrates both the effectiveness and tolerability of multimodal treatment with SA peels and PDL for the treatment of acne. This combined approach should be strongly considered as a treatment modality for patients who are not responding to conventional methods of treatment, who are non-compliant with oral or topical medications, or for those who prefer in-office physician controlled treatments.

One limitation to the study could have been the modest sample size, though statistical significance was appreciated between the two treatment arms of the study. In the future, clinical studies could be designed to evaluate the two treatment arms on a larger population size. Additionally, patients could be followed for a longer duration after their final treatment to assess duration of efficacy of SA peels and PDL therapy when used in conjunction.

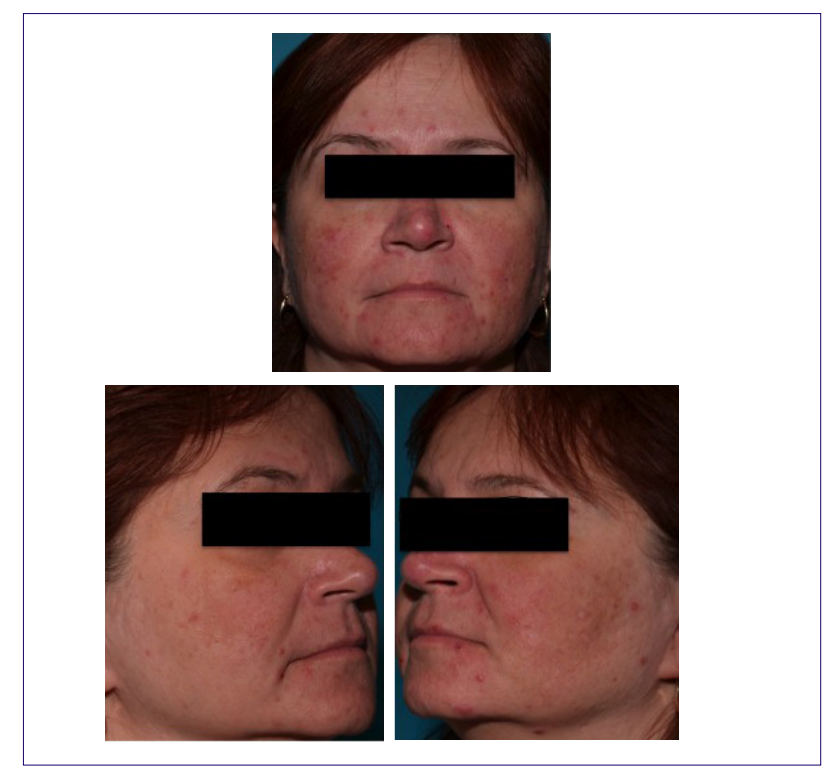

Figure 2. Patient progress from week 0 to week 9 . Patient is pictured at week 0 (above). At week 9 , the side of face treated with the combined PDL and SA treatment is shown on the bottom left, and the side of the face treated with the SA peel alone is shown on the bottom right. The DLQI questionnaire yielded a statistically significant decrease in level of self-consciousness about appearance between week 0 and week $9(P=0.038)$. There were no reported adverse or serious adverse events in either group. 


\section{Conclusion}

Acne subjects had significant benefit from the peel alone, but had greater significant benefit from PDL treatment used in conjunction with SA peels. The addition of PDL to SA peel therapy leads to better outcomes in acne management and reduces adverse psychological consequences.

\section{Acknowledgements}

We would like to thank Dr. James Sinacore, PhD for his help with our statistical analyses.

\section{Conflict of Interest}

All authors report no conflicts of interest. No financial support was provided for the production of this manuscript.

\section{References}

1. Zaenglein AL, Graber EM, Thiboutot DM. Acne Vulgaris and Acneiform Eruptions. In: Goldsmith LA, Katz SI, Gilchrest BA, Paller AS, Leffell DJ, Wolff K. eds. Fitzpatrick's Dermatology in General Medicine. 8th ed. New York, NY: McGraw-Hill; 2012.

2. Jowett S, Ryan T. Skin disease and handicap: an analysis of the impact of skin conditions. Soc Sci Med. 1985;20(4):425-429. doi:10.1016/02779536(85)90021-8

3. Gupta MA, Gupta AK. Depression and suicidal ideation in dermatology patients with acne, alopecia areata, atopic dermatitis and psoriasis. $\mathrm{Br}$ J Dermatol. 1998;139:846-850. doi:10.1046/j.13652133.1998.02511.x

4. Williams HC, Dellavalle RP. Acne Vulgaris. Lancet. 2012;379(9813):361-372. doi:10.1016/s0140 6736(11)60321-8

5. Haider A, Shaw JC. Treatment of Acne Vulgaris. JAMA. 2004;292(6):726-735. doi:10.1001/jama.292.6.726

6. Tan JK. Psychosocial Impact of Acne Vulgaris: Evaluating the Evidence. Skin Ther Lett. 2004;9(7):1-3.

7. Strauss JS, Krowchuk DP, Leyden JJ, et al. Guidelines of care for acne vulgaris management. J Amn Acad Dermatol. 2007;56(4):651-663.

8. Kim RH, Armstrong AW. Current state of acne treatment: highlighting lasers, photodynamic therapy, and chemical peels. Dermatol Online J. 2011;17(3):2.

9. Witten C. Department of Health and Human Services Re: K043251 Section 510(k) Premarket Notification 2005. Food and Drug Administration; 2014.

10. Boon LM, Vikkula M. Vascular Malformations. In: Goldsmith LA, Katz SI, Gilchrest BA, Paller AS, Leffell DJ, Wolff K, eds. Fitzpatrick's Dermatology in General Medicine. 8th ed. New York, NY: McGraw-Hill, 2012.

11. Leheta TM. Role of the 585-nm pulsed dye laser in the treatment of acne in comparison with other topical therapeutic modalities. J Cosmet Laser Ther. 2009;11:118-124. doi:10.1080/14764170902741329

12. Seaton ED, Mouser PE, Charakida A, Alam S, Seldon $\mathrm{PM}$, Chu AC. Investigation of the mechanism of action of nonablative pulsed-dye laser therapy in photorejuvenation and inflammatory acne vulgaris. $\mathrm{Br}$
J Dermatol. 2006;155(4):748-755. doi:10.1111/j.13652133.2006.07429.x

13. Jih $\mathrm{MH}$, Kimyai-Asadi A. Laser Treatment of Acne Vulgaris. Semin Plast Surg. 2007;21(3):167-174.

14. Orringer JS, Sachs DL, Bailey E, Kang S, Hamilton T, Voorhees JJ. Photodynamic therapy for acne vulgaris: a randomized, controlled, split-face clinical trial of topical aminolevulinic acid and pulsed dye laser therapy. J Cosmet Dermatol. 2010;9(1):28-34. doi:10.1111/j.1473-2165.2010.00483.x

15. Kessler E, Flanagan K, Chia C, Rogers C, Glaser DA. Comparison of alpha- and beta-hydroxy acid chemical peels in the treatment of mild to moderately severe facial acne vulgaris. Dermatol Surg. 2008;34(1):45-50.

16. Rendon MI, Berson DS, Wang B. Evidence and Considerations in the Application of Chemical Peels in Skin Disorders and Aesthetic Resurfacing. J Clin Aesthet Dermatol. 2010;3(7):32-43.

17. Burkhart CN. Katz KA. Other Topical Medications. In: Goldsmith LA, Katz SI, Gilchrest BA, Paller AS, Leffell DJ, Wolff K, eds. Fitzpatrick's Dermatology in General Medicine. 8th ed. New York, NY: McGraw-Hill, 2012.

18. Lin AN, Nakatsui T. Salicylic acid revisited. Int $J$ Dermatol. 1998;37:335-342. doi:10.1046/j.13654362.1998.00452.x

19. Lee HS, Kim IH. Salicylic acid peels for the treatment of acne vulgaris in Asian patients. Dermatol Surg. 2003;29(12):1196-1199. doi:10.1111/j.15244725.2003.29384.x

20. Dreno B, Poli F, Pawin H, et al. Development and evaluation of a Global Acne Severity Scale (GEA Scale) suitable for France and Europe. J Eur Acad Dermatol Venereol. 2011;25(1):43-48. doi:10.1111/j.14683083.2010.03685.x

21. Basra MK, Fenech R, Gatt RM, Salek MS, Finlay AY. The Dermatology Life Quality Index 19942007: a comprehensive review of validation data and clinical results. Br J Dermatol. 2008;159(5):997-1035. doi:10.1111/j.1365-2133.2008.08832.x

22. Haedersdal M, Togsverd-Bo K, Wulf HC. Evidencebased review of lasers, light sources and photodynamic therapy in the treatment of acne vulgaris. J Eur Acad Dermatol Venereol. 2008;22(3):267-278. doi:10.1111/ j.1468-3083.2007.02503.x

23. Orringer JS, Kang S, Hamilton T, et al. Treatment of acne vulgaris with a pulsed dye laser: a randomized controlled trial. JAMA. 2004;291(23):2834-2839. doi:10.1001/jama.291.23.2834

24. Seaton ED, Charakida A, Mouser PE, Grace I, Clement RM, Chu AC. Pulsed-dye laser treatment for inflammatory acne vulgaris: randomized controlled trial. Lancet. 2003;362(9393):1347-1352. doi:10.1016/ s0140-6736(03)14629-6

25. Jung JY, Choi YS, Yoon MY, Min SU, Suh DH. Comparison of a pulsed dye laser and a combined 585/1,064-nm laser in the treatment of acne vulgaris. Dermatol Surg. 2009;35(8):1181-1187. doi:10.1111/ j.1524-4725.2008.34427.x 